\title{
Optimization of Low-Dose CT Protocol in Pediatric Nuclear Medicine Imaging
}

Hanna Piwowarska-Bilska ${ }^{1}$, Leszek J. Hahn², Bozena Birkenfeld ${ }^{1}$, Katarzyna Cichon-Bankowska ${ }^{1}$, Maria H. Listewnik ${ }^{1}$, and Piotr Zorga ${ }^{1}$

${ }^{1}$ Department of Nuclear Medicine, Pomeranian Medical University, Szczecin, Poland; and ${ }^{2}$ Department of Diagnostic Imaging, Foothills Medical Centre, Calgary, Alberta, Canada

This study was performed to find the optimal low-dose CT protocol for children being imaged on SPECT/CT scanners not equipped with automatic dose control. For SPECT/CT systems with manually adjustable $x$-ray tube voltage $(\mathrm{kV})$ and anode current $(\mathrm{mA})$, an optimized protocol makes it possible to minimize the dose to patients. Methods: Using the 4-slice low-dose CT component of a commercially available SPECT/ CT scanner, we compared the signals reaching the CT detector after radiation passes through objects of different sizes. First, the exit dose rates were measured for combinations of available voltages and currents. Next, imaging parameters were selected on the basis of acceptable levels of exit dose rates, cylindric phantoms of different diameters approximating children of different sizes were scanned using these parameters, and the quality of the CT images was evaluated. Finally, weighted CT dose indexes for abdomen and head CT dose phantoms simulating, respectively, adult and pediatric patients were measured using exactly the same techniques to estimate and compare doses to these 2 groups of patients. Results: For children with torsos smaller than $150 \mathrm{~mm}$, imaging can be performed using the lowest available voltage and current $(120 \mathrm{kV}$ and $1 \mathrm{~mA}$, respectively). For children with torsos less than $250 \mathrm{~mm}, 140 \mathrm{kV}$ and $1.5 \mathrm{~mA}$ can be used. For patients with torsos greater than 250 and less than $300 \mathrm{~mm}, 140 \mathrm{kV}$ and $2 \mathrm{~mA}$ can be used. Regarding the signal-to-noise ratio, all these parameters give an excellent signal and fully acceptable noise levels. Conclusion: For the SPECT/CT system studied, even the lowest available voltage and current used for scanning pediatric patients did not cause signal-to-noise degradation, and the use of these settings substantially lowered the dose to the patients.

Key Words: low-dose CT; SPECT/CT; pediatric radiation exposure; CT dose optimization

J Nucl Med Technol 2010; 38:181-185

DOI: 10.2967/jnmt.109.073486

D iagnostic SPECT/CT systems allow CT scans to be registered with nuclear medicine scans so that radioisotope

Received Dec. 30, 2009; revision accepted Jul. 2, 2010.

For correspondence or reprints contact: Hanna Piwowarska-Bilska, Department of Nuclear Medicine, Pomeranian Medical University, ul. Unii Lubelskiej 1, 72-252 Szczecin, Poland.

E-mail: hannap@pam.edu.pl

COPYRIGHT ( 2010 by the Society of Nuclear Medicine, Inc. uptake can be localized. CT scans are additionally used for attenuation correction of radioisotope images. However, compared with SPECT-only examinations, SPECT/CT examinations increase the radiation exposure of patients. SPECT/CT systems with adjustable $\mathrm{x}$-ray tube voltage $(\mathrm{kV})$ and anode current $(\mathrm{mA})$ make it possible to minimize the dose to patients. Reasonable reduction of CT-related dose in individual patients is an important issue, particularly in children $(1,2)$. The aim of this study was to find the optimal pediatric CT protocol that reduces radiation risk.

\section{MATERIALS AND METHODS}

All investigations used the 4-slice low-dose CT component of the Infinia Hawkeye 4 SPECT/CT system (GE Healthcare). In this system, both the voltage and the current are adjustable. To compare the signals reaching the CT detector after radiation passes through objects of different sizes, we measured the exit dose for all combinations of available voltages and currents. To simulate patients of different sizes, we used phantoms containing various volumes of water. During all measurements, the x-ray tube was immobilized at the 12-o'clock position in the scanner service mode. To validate this approach and to find the relationship between exit dose rates (detector signal) and CT image quality, we scanned 4 cylindric phantoms filled with water. For this purpose, the system was switched from stationary to tomographic mode, and noise levels representing signalto-noise ratios in CT images of these phantoms were measured. The diameters of these phantoms corresponded to children of different weights and sizes. To estimate the doses to large and small patients imaged at variable voltages and currents, we scanned the abdomen and head phantoms and measured the weighted CT dose index $\left(\mathrm{CTDI}_{\mathrm{w}}\right)$.

\section{Measurement of Exit Dose}

Radiation exiting the water phantom and reaching the CT detector was measured using a Dosemeter/Ratemeter 2026C (Radcal Corp.) ionization chamber, 20X6-3. For this purpose, 8 combinations of available voltage (120 or $140 \mathrm{kV})$ and current $(1,1.5,2$, or $2.5 \mathrm{~mA})$ were used at a constant time of $23 \mathrm{~s}$, which corresponds to the time of full gantry rotation in the $\mathrm{CT}$ mode of scanning. To estimate the strength of the signal reaching the CT detectors, we 
calculated dose rates by dividing the measured cumulative dose by that time. An acrylic water-filled cylinder was used as a phantom simulating attenuation by the human body. The cylinder was $330 \mathrm{~mm}$ in height and $210 \mathrm{~mm}$ in diameter. The cylinder was placed on the patient table, vertically (Fig. 1), with its center coinciding with the geometric center of the x-ray fanbeam. The ionization chamber was positioned directly under the cylinder at its center. The water level in the cylinder was increased in steps of $50 \mathrm{~mm}$, from $0 \mathrm{~mm}$ to a maximum of $300 \mathrm{~mm}$. All these measurements were performed twice to check repeatability. Overall, 168 measurements of exit dose were performed under the unchanged geometry.

\section{Noise Level Evaluation}

Noise levels for calculation of signal-to-noise ratios (3) were evaluated by imaging sets of water-filled cylinders 270, 200, 160, and $100 \mathrm{~mm}$ in diameter in SPECT/CT mode using a bone SPECT protocol. Voltage and current were identical to those used for the exit dose measurements. The reference to which the data obtained from all scanned cylinders were compared corresponded to an adult patient, as represented by a Data Spectrum torso anthropomorphic phantom. The water-filled part of this phantom was scanned at $140 \mathrm{kV}$ and $2.5 \mathrm{~mA}$, and the time of rotation was $23 \mathrm{~s}$. It was assumed that these parameters were tested in the factory and then selected as optimal for adult patient imaging. The slice thickness and collimation are not adjustable for this SPECT/CT system and for all scans were 5 and $20 \mathrm{~mm}$, respectively. For data analysis, circular regions of interest were used. One large region of interest covering about $80 \%$

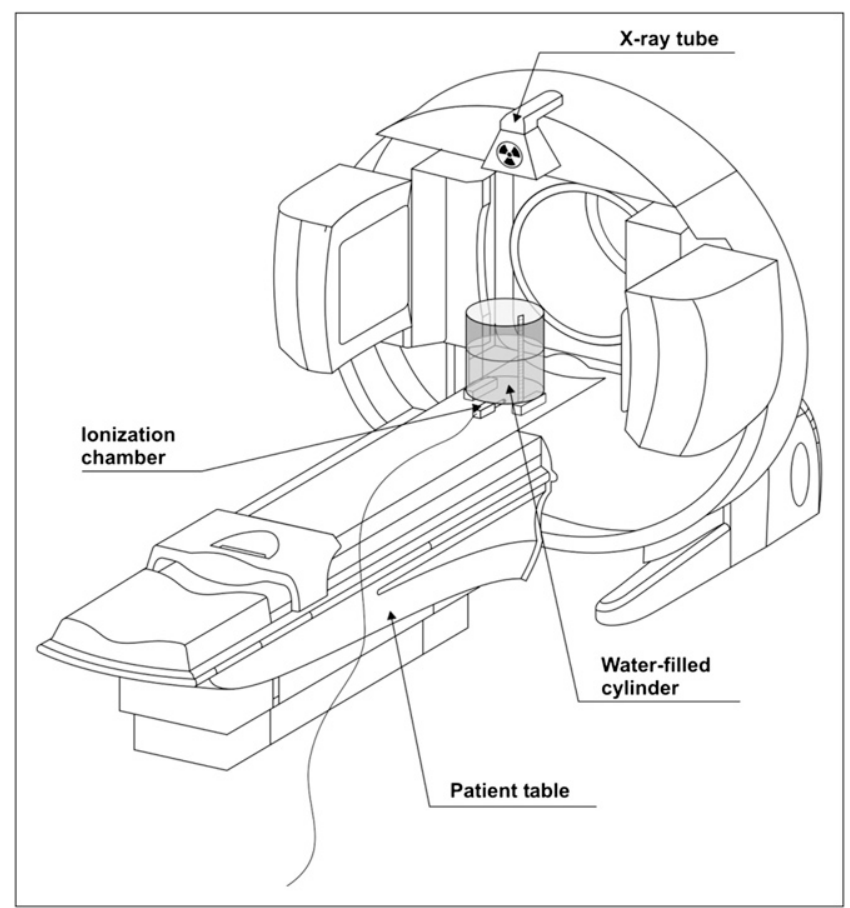

FIGURE 1. Schematic illustration of exit dose measurement geometry. of the image of a scanned cylinder was used to calculate the average SD in the image. This SD represented the noise level for a particular set of parameters and cylinder scan. To evaluate the range of SDs in different parts of the CT image, the set of smaller regions of interest with a diameter equal to about $40 \mathrm{~mm}$ was placed circumferentially (12-, 3-, 6-, and 9-o'clock positions) and along the $x$ - and $y$-axes of CT images of cylinders and torso phantom. SDs in selected regions of interest for a particular slice were calculated using the system software and recorded from a region-ofinterest statistics database.

\section{Measurements of $\mathrm{CTDI}_{\mathrm{w}}$}

The most commonly used dose descriptor is CT dose index. The $\mathrm{CTDI}_{\mathrm{w}}$, measured at the center and periphery of CT dose index phantoms, provides a convenient estimate of patient dose for a procedure $(4,5)$. $\mathrm{CTDI}_{\mathrm{w}}$ is defined as follows:

$$
\mathrm{CTDI}_{\mathrm{w}}=(1 / 3)\left(\mathrm{CTDI}_{100}\right)_{\text {center }}+(2 / 3)\left(\mathrm{CTDI}_{100}\right)_{\text {periphery }} \text {. Eq. } 1
$$

$\mathrm{CTDI}_{100}$ is the integral of the dose profile along a line perpendicular to the tomographic plane divided by the product of the nominal tomographic section thickness and the number of tomograms produced in a single axial scan. Because the ionization chamber measures an integrated exposure along its $100-\mathrm{mm}$ length, $\mathrm{CTDI}_{100}$ can be calculated according to the following formula:

$$
\mathrm{CTDI}_{100}=(f \cdot E)(L / T) .
$$

Eq. 2

$f$ is the conversion factor from exposure ( $\mathrm{mR}$ ) to a dose in air (mGy), $E$ is the measured value of exposure, $L$ is the active length of the pencil ionization chamber, and $T$ is the nominal width of each acquired section.

For CT dose index measurements, a CT dose probe was used: a 10X5-3CT pencil-type ionization chamber with a sensitive length of $100 \mathrm{~mm}$ (Radcal). Two phantoms were used: solid polymethylmethacrylate disks measuring 160 $\mathrm{mm}$ in diameter (head phantom simulating a child's body) and $320 \mathrm{~mm}$ in diameter (abdomen phantom simulating an adult's body), both $140 \mathrm{~mm}$ thick. There are 5 holes in each of the phantoms, 4 circumferential $(4 \mathrm{~mm}$ from the edge) and 1 central, with acrylic rods to plug the holes when not in use. The holes are $13.1 \mathrm{~mm}$ in diameter to accommodate the size of a standard CT probe. Because the main aim of this work was to evaluate the dose reduction when small patients (children) are scanned using the lowest available current and voltage, only 2 sets of scanning parameters were tested. First, $140 \mathrm{kV}$ and $2.5 \mathrm{~mA}$ (maximal available values) were used for scanning the abdomen and head phantoms. These parameters would correspond to the use of a nonadjustable scanner, independent of a patient's size. Then, the lowest available voltage $(120 \mathrm{kV})$ and current $(1 \mathrm{~mA})$ were used for scanning the head phantom only, which would correspond to a scanner that is adjustable according to the weight or size of smaller patients. 
TABLE 1

Average Exit Dose Rates

\begin{tabular}{|c|c|c|c|c|c|c|c|c|}
\hline \multirow[b]{2}{*}{ Voltage (kV) } & \multirow[b]{2}{*}{ Current $(\mathrm{mA})$} & \multicolumn{7}{|c|}{ Exit dose rate $\left(10^{-2} \mu \mathrm{Gy} / \mathrm{s}\right)^{\star}$} \\
\hline & & $0 \mathrm{~mm}$ & $50 \mathrm{~mm}$ & $100 \mathrm{~mm}$ & $150 \mathrm{~mm}$ & $200 \mathrm{~mm}$ & $250 \mathrm{~mm}$ & $300 \mathrm{~mm}$ \\
\hline 140 & 2.5 & 2,138 & 1,100 & 522 & 235 & 110 & 48 & 24 \\
\hline 140 & 2.0 & 1,705 & 887 & 426 & 193 & 87 & 39 & 18 \\
\hline 140 & 1.5 & 1,295 & 675 & 323 & 148 & 67 & 29 & 15 \\
\hline 140 & 1.0 & 883 & 460 & 218 & 105 & 44 & 20 & 9 \\
\hline 120 & 2.5 & 1,396 & 713 & 332 & 147 & 65 & 29 & 13 \\
\hline 120 & 2.0 & 1,126 & 574 & 264 & 117 & 53 & 23 & 10 \\
\hline 120 & 1.5 & 853 & 417 & 201 & 87 & 40 & 17 & 7 \\
\hline 120 & 1.0 & 580 & 280 & 140 & 61 & 28 & 12 & 5 \\
\hline
\end{tabular}

${ }^{*}$ Data are for the stated different depths of water in a cylindric phantom simulating attenuation in a patient. Rates are calculated by dividing cumulative exit doses by time of measurement.

\section{RESULTS}

Table 1 shows the exit dose rates. These calculations used the arithmetic average of 3 cumulative exit doses measured with the 20X6-3 ionization chamber. Because the dispersion of measured cumulative exit doses was small (coefficient of variation $<1 \%$ ), it is not shown in Table 1 . Figure 2 is the graphic representation of the results in Table 1. The shaded area represents parameters giving a detector signal equal to or higher than that produced using the maximum voltage and current available. Table 2 shows noise levels in CT images of cylindric phantoms of different diameters. They represent children of varying sizes scanned using different parameters. These measurements helped to justify the validity of using the exit dose values as a basis for selecting parameters adequate for a child's size.

$\mathrm{CTDI}_{\mathrm{w}}$ results for both CT dose phantoms are shown in Table 3.

Table 4 contains CT acquisition protocols proposed for technologists performing patient studies on the Infinia Hawkeye 4. The information on weight, age, and corresponding patient dimensions is based on the available literature (6). In this study, the arithmetic mean of the chest and abdomen dimensions was used as the average patient size because all measurements of CT dose index and noise were

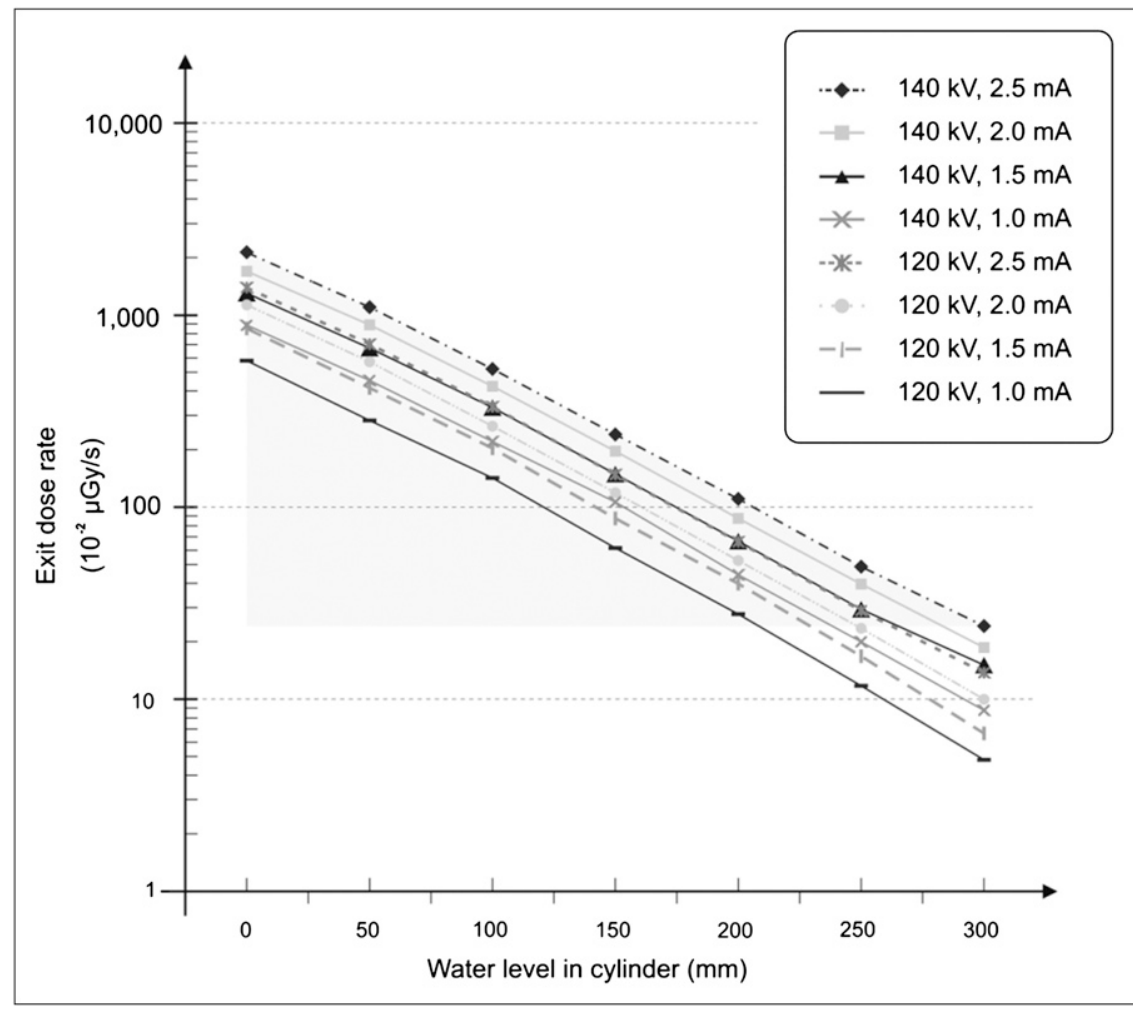

FIGURE 2. Semilogarithmic graph of exit dose rates for various voltages and currents vs. increasing depth of water in phantom simulating attenuation in patient. 
TABLE 2

Signal-to-Noise Results for CT Images of Water Phantoms

\begin{tabular}{|c|c|c|c|c|c|c|}
\hline \multirow[b]{2}{*}{ Voltage (kV) } & \multirow[b]{2}{*}{ Current (mA) } & \multirow[b]{2}{*}{ Reference phantom* } & \multicolumn{4}{|c|}{ Phantom diameter (mm) } \\
\hline & & & 270 & 200 & 160 & 100 \\
\hline 140 & 2.5 & $42(35-52)$ & 27 (23-34) & $18(15-21)$ & $11(9-14)$ & \\
\hline 140 & 2.0 & & $30(27-35)$ & $21(22-25)$ & $12(10-15)$ & \\
\hline 140 & 1.5 & & $35(26-45)$ & $25(23-32)$ & $14(12-16)$ & \\
\hline 140 & 1.0 & & $43(36-54)$ & $29(28-33)$ & $16(14-19)$ & \\
\hline 120 & 2.5 & & $33(27-43)$ & $22(21-26)$ & $13(11-16)$ & \\
\hline 120 & 2.0 & & $37(25-49)$ & $25(24-31)$ & $15(13-16)$ & \\
\hline 120 & 1.5 & & $43(37-52)$ & $29(27-34)$ & $17(15-19)$ & \\
\hline 120 & 1.0 & & $53(44-69)$ & $36(33-42)$ & $20(17-23)$ & $11(10-12)$ \\
\hline
\end{tabular}

performed on cylindric phantoms. The relationship between body size and weight for adolescents was extrapolated from the data on children and adult patients because of a lack of relevant data in the literature. The results from Table 1 and information about the relationship between children's ages, weights, and sizes were the basis for the proposed low-dose CT acquisition protocols.

\section{DISCUSSION}

The results of this study indicate the possibility of substantially decreasing doses to patients without losing the detector signal. Thus, there is a chance that the risk in children imaged with SPECT/CT systems can be reduced. Table 1 shows that for water layers thinner than $200 \mathrm{~mm}$, the exit dose measured at the minimum available voltage and current $(120 \mathrm{kV}, 1 \mathrm{~mA}$, respectively) is stronger than that detected when a $300-\mathrm{mm}$-thick layer of water is penetrated by radiation generated using maximum voltage and current (140 kV and $2.5 \mathrm{~mA}$, respectively). Drawing a line parallel to the "water level in cylinder" axis to intersect the "object exit dose" axis at a point corresponding to the detected exit dose when using $140 \mathrm{kV}$ and $2.5 \mathrm{~mA}$ for objects $300 \mathrm{~mm}$ in diameter (which corresponds to penetration of a 300-mm-thick water layer), one can find all the parameters for all object diameters that give a signal equal to or greater than this first one (dashed area in Fig. 2). On this basis, parameters optimizing doses to patients of different sizes can be selected. Thus, for infants $150 \mathrm{~mm}$ in diameter or smaller, the technique utilizing the lowest available voltage and current can be used because it still gives an excellent detector signal. For patients greater than $150 \mathrm{~mm}$ and up to $200 \mathrm{~mm}$ in diameter, $120 \mathrm{kV}$ and $2 \mathrm{~mA}$ are sufficient. Patients greater than $200 \mathrm{~mm}$ and up to $250 \mathrm{~mm}$ can be scanned at $140 \mathrm{kV}$ and $1.5 \mathrm{~mA}$. For patients from 250 to $300 \mathrm{~mm}$ (adolescents), scanning at $140 \mathrm{kV}$ and $2 \mathrm{~mA}$ is suitable. The fact that these parameters translate into a substantial reduction in doses to children and smaller patients is an important message to users of SPECT/CT systems with adjustable settings for voltage and current.

The signal-to-noise results for scanning various sizes of water-filled phantoms with various parameters (Table 2) supports the exit-dose-level criterion as a basis for selecting the imaging parameters. The noise level in the reference image (torso phantom) averaged 42 and ranged from 35 (minimum, the best) to 52 (maximum, the worst). This level is comparable to results obtained for the phantom with a diameter of $270 \mathrm{~mm}$ (the largest) scanned at $140 \mathrm{kV}$ and $1 \mathrm{~mA}$ : the noise level in that case averaged 43 and ranged from 36 to 54 . The noise levels for all remaining phantoms scanned, even at $120 \mathrm{kV}$ and $1 \mathrm{~mA}$, were better than the reference level. Additionally, comparison with the data from Table 1 and Figure 2 shows that using the exit dose rate as a basis for selection of scanning parameters is actually more restrictive than would be selection based on analysis of noise levels only.

The dose reduction can clearly be seen from the $\mathrm{CTDI}_{\mathrm{w}}$ results for the various scanning parameters and for the 2 phantoms (Table 3). When one switches from $140 \mathrm{kV}$ and $2.5 \mathrm{~mA}$ to $120 \mathrm{kV}$ and $1 \mathrm{~mA}$ for children $150 \mathrm{~mm}$ in diameter, the dose decreases from $7.23 \mathrm{mGy}$ to $2.01 \mathrm{mGy}$. For

TABLE 3

$\mathrm{CTDI}_{\mathrm{W}}$ for Various Scanning Parameters and Phantoms

\begin{tabular}{ccc}
\hline CT parameters & CTDI $_{\mathrm{w}}$ for abdomen phantom (mGy) & CTDI $_{\mathrm{w}}$ for head phantom (mGy) \\
\hline $\begin{array}{l}120 \mathrm{kV} ; 1 \mathrm{~mA} \text {; axial scanning; collimation, } 20 \mathrm{~mm} ; \\
\text { slice thickness, } 5 \mathrm{~mm}\end{array}$ & - & 2.01 \\
$\begin{array}{r}140 \mathrm{kV} ; 2.5 \mathrm{~mA} \text {; axial scanning; collimation, } 20 \mathrm{~mm} ; \\
\text { slice thickness, } 5 \mathrm{~mm}\end{array}$ & 5.42 & 7.23 \\
\hline
\end{tabular}


TABLE 4

Proposed CT Protocol for SPECT/CT in Children

\begin{tabular}{l}
\hline $\begin{array}{c}\text { Relationship between } \\
\text { patient average } \\
\text { diameter }{ }^{*} \text { and } \\
\text { weight }(\mathrm{mm} \rightarrow \mathrm{kg})\end{array}$ \\
$\begin{array}{c}\text { Patient } \\
\text { weight }(\mathrm{kg})\end{array}$
\end{tabular}

infants smaller than $150 \mathrm{~mm}$, the dose reduction would be even greater.

The results presented in this article should be instructive for producers of cheaper SPECT/CT systems in which the CT component is not equipped with automatic dose control. There is definitely some room for improvement. For imaging of small children, the mAs - as a combination of changes in $\mathrm{kV}, \mathrm{mA}$, and times of data acquisition - can be decreased further. The $\mathrm{CTDI}_{\mathrm{w}}$ is 3.6-fold higher when the parameters are not adjustable than when the parameters can be properly adjusted to the weight and height of the patient. Table 4 presents our proposed protocol for the CT part of the acquisition on an Infinia Hawkeye 4. For clinical acquisitions, technologists can set the optimal current and voltage based on patient size and weight.

\section{CONCLUSION}

The radiation dose to children imaged with low-dose CT as part of SPECT/CT studies can be reduced. Proper reduction of the voltage and current of the Infinia Hawkeye 4 for patients of smaller size and weight does not decrease the signal strength reaching the CT detectors and does not affect image quality.

\section{REFERENCES}

1. Committee to Assess Health Risks from Exposure to Low Levels of Ionizing Radiation, National Research Council of the National Academies. Health Risks from Exposure to Low Levels of Ionizing Radiation: BEIR VII, Phase 2. Washington, DC: National Academies Press; 2006.

2. Brenner DJ, Hall E. Computed tomography: an increasing source of radiation exposure. N Engl J Med. 2007;357:2277-2284.

3. Sprawls P. CT image detail and noise. Radiographics. 1992;12:1041-1046.

4. Bongartz G, Golding SJ, Jurik AG, et al. European Guidelines for Quality Criteria for Computed Tomography: EUR 16262. Available at: http://www.drs.dk/ guidelines/ct/quality/. Accessed August 30, 2010.

5. Shope TB, Gagne RM, Johnson GC. A method for describing the doses delivered by transmission x-ray tomography. Med Phys. 1981;8:488-495.

6. Kereiakes GJ, Rosenstein M. Handbook of Radiation Doses in Nuclear Medicine and Diagnostic X-Ray. Boca Raton, FL: CRC Press; 1980:187, 214. 